\title{
Design and numerical simulation of an immnuunoisolation perfusion co-culture bioreactor for expansion of NSCs
}

Kedong SONG ${ }^{1, a, \star}$, Siyu JIANG ${ }^{1, b}$, Jiaxin $\operatorname{TIAN}^{1, \mathrm{c}}$, Zeren $\mathrm{JIAO}^{1, \mathrm{~d}}$, Yiwei WANG $^{2, e}$, Jiaquan ZHAO ${ }^{3, \mathrm{f}, *}$, Tianqing $\mathrm{LIU}^{1, \mathrm{~g}, *}$

${ }^{1}$ State Key Laboratory of Fine Chemicals, Dalian R\&D Center for Stem Cell and Tissue Engineering, Dalian University of Technology, Dalian 116024, China

${ }^{2}$ Burns Research Group, ANZAC Research Institute, University of Sydney, Concord, NSW, 2139, Australia,

${ }^{3}$ School of Energy and Power Engineering, Huazhong University of Science and Technology, Wuhan 430074, China

aemail: kedongsong@dlut.edu.cn, bemail: syjiang0226@mail.dlut.edu.cn, cemail:

1219589234@qq.com, demail: jiaozeren@mail.dlut.edu.cn, eemail:yi.w.wang@sydney.edu.au, ${ }^{1 f} \mathrm{em}$ ail:jiaquan_zhao@hust.edu.cn, ${ }^{9}$ email: liutq@dlut.edu.cn

Keywords: Immnuoisolation; Perfusion; NSCs; Expansion; Co-culture

\begin{abstract}
Considering the immnuoisolation from the neural stem cells (NSCs) and endothelial progenitor cells (EPCs), the requirements of the carrier, shear tolerance and other factors, this study designed an immunoisolation perfusion co-culture bioreactor system, by dynamic perfusion culture environment of its upper and lower bi-levels and the immnuoisolation of intermediate polycarbonate membrane, combining the measure of embedding NSCs and EPCs with calcium alginate/gelatin microbeads, integrating hypoxic environment effectively to achieve the expansion of NSCs. The results show that the microbeads are more evenly distributed under dynamic perfusion conditions, the velocity of medium is slightly larger near polycarbonate membrane and the culture chamber wall surface, and has more balanced distribution of other regions, static pressure and dynamic pressure of the culture chamber are generally evenly distributed. Studies showed that the expansion of NSCs under perfusion conditions, is able to meet the mass transfer and provide a stable environment to the neural stem cell expansion.
\end{abstract}

\section{Introduction}

Neural stem cells (NSCs) have self-renewal, replication capacity and multi-differentiation potential. They can differentiate into neurons, astrocytes and oligodendrocytes, which can be applied to the treatment of Parkinson's disease, spinal cord injury, cerebrovascular disease, etc [1-3]. At present, the application and research of NSCs include direct use of cell transplantation for replacement therapy, using NSCs as gene carrier and carrying the target gene with therapeutic action and self-repair of NSCs induced to self-differentiation, etc [4-6]. At the same time, with the continuous further research of the human nervous system and the rapid development of clinical cell therapy, the quantity and quality of NSCs have been put forward higher requirements. Therefore, how to harvest a sufficient number of NSCs on the basis of effectively maintaining the stemness, is the key issue to be resolved.

The main methods of in vitro culture of NSCs were the cultivation of the sphere in the culture vessel by suspension method and the culture on the surface of the treated culture environment in a single wall $[7,8]$. The culture way of NSC sphere is simple to operate, in which cell number is relatively large. NSC spheres can be blown about and differentiate into specific cells through different induced growth factors. But the shortcoming is, with the increase of the sphere size, the requirement of mass transfer cannot be satisfied. So the dark core was formed inside the sphere, where apoptotic, necrotic, and phagocytic cells were easily appeared, and perhaps even the hollow 
of the NSC spheres [9]. Monolayer adhesion method can effectively meet the requirements of material transfer. However, the cell adhesion area is small in this way, which limits the increment of cells, and is therefore not conducive to the cell expansion. Simultaneously, if the cells are directly exposed to the dynamic liquid environment, the shear stress will cause damage to NSCs, resulting in decreased activity, and even cell death [10].

The NSCs were cultured in a suitable bioreactor system with an ideal three-dimensional (3D) cell carrier, which can satisfactorily solved the problem above mentioned. The ideal 3D cell carrier can provide attachment sites for NSCs, and allow timely diffusion of nutrients and metabolites. It is easy to set up connections between cells, can make NSCs a suitable spatial distribution and cell connections, and also provide specific growth and differentiation signals for cells. In addition, the cell carrier can prevent NSCs from being more sensitive to the shear force in dynamic environment, which has a very good protection effect. The suitable dynamic bioreactor system can not only provide enough residence time for the internal culture of NSCs, effectively meet the requirements of material delivery, but also achieve the effective isolation of stromal cells in co-culture system $[11,12]$.

Endothelial progenitor cells (EPCs) have the function of mobilization, proliferation, migration and homing to sites of injury and differentiation to vascular endothelial cells in the effect of specific factors, which make it available to be used for co-culture with NSCs. Research shows that $[13,14]$, neurogenesis occurs commonly after the angiogenesis. Neurogenesis after injury phenomenon is also appearing along the vessel path with a large number of vascular endothelial differentiation. Angiogenesis provides a good platform for neurogenesis. And EPCs also showed a significant neuroprotective effect. Therefore, neural microenvironment formed by co-culture of EPCs and NSCs can promote the proliferation of NSCs to a large extent; In addition, EPCs can delay the differentiation of NSCs by secreting cytokines such as VEGF and FGF-b, etc [14]. Therefore, if the co-culture was carried out using EPCs and NSCs, it is expected to achieve large scale expansion of NSCs on the basis of effectively maintaining NSCs dryness.

Based on this, we designed an immunoisolation perfusion co-culture bioreactor (IPCB), which is used by two kinds of different cells in the dynamic perfusion environment with up and down bi-level culture chamber, and the numerical simulation and flow field analysis of IPCB were also carried out.

\section{Software and methods}

\section{Gambit and Fluent}

ANSYS Fluent software (ANSYS, Canonsburg, PA, USA) can be used to realize the mass transfer in a bioreactor culture system. It provides ideal grid adaptability and can solve complex fluid problems easily by an irregular grid, which is provided by complex geometry. In view of the characteristics of every physical problem in fluid dynamics, a suitable numerical solution is provided by ANSYS Fluent in order to achieve optimality in multiple aspects such as computation speed, stability, and precision.

On the basis of computational fluid mechanics principle, with the help of fluent software, the mass transfer analysis in an IPCB provides important theoretical and reference data for the subsequent cell and tissue experiments, to make it more effective application in the 3D fabrication of tissue engineered cartilages/bones.

Physical and simplified model of bioreactor flow

To simplify the calculation, the flow of the static environment was treated as a two-dimensional problem. As the rotation speed of the IPCB used in further fabrication experiment was quite low, the calculated speed of the IPCB was therefore set at $50 \mathrm{rpm}$, and the flow field in IPCB with this rotating speed still remained laminar flow. Therefore, laminar modeling of Fluent could be used for numerical simulation calculations.

Based on this, mass fraction distribution of microspheres constructs in an IPCB during the culture process was calculated. The specific conditions and calculation objects were as follows: in order to calculate the force condition and its distribution around the flow field of the microspheres 
constructs, the three-dimensional section size of the constructs was set to a cane with a middle straight height of $5 \mathrm{~mm}$, dimension of $70 \mathrm{~mm}$ and cane angle of $60^{\circ}$. Changes in the simulation calculations, collected from different section, were analyzed when the rotation direction and speed of the IPCB was determined. The partition of the 3D mesh and constraints that defined the physical boundaries above were shown in Table.1\&2

Table 1 Parameters of bioreactor

\begin{tabular}{cc}
\hline Dimension $(\mathrm{mm})$ & 70 \\
\hline Height $(\mathrm{mm})$ & 5 \\
$\begin{array}{c}\text { Cone angle }\left({ }^{\circ}\right) \\
\text { Dimension of entrance } \\
(\mathrm{mm})\end{array}$ & 60 \\
$\begin{array}{c}\text { Dimension of outlet } \\
(\mathrm{mm})\end{array}$ & 5 \\
\hline
\end{tabular}

Table 2 Parameters and boundary conditions

\begin{tabular}{cc}
\hline Culture medium density $\left(\mathrm{kg} / \mathrm{m}^{3}\right)$ & 1050 \\
\hline Dynamic viscosity $(\mathrm{Pa} . \mathrm{s})$ & $3 \times 10^{-3}$ \\
Vessel rotational velocity $(\mathrm{rpm})$ & 50 \\
Fluent version & $3 \mathrm{~d}$ \\
Solver & Segregated, implicit \\
Pressure & Body-force weighted \\
Pressure-velocity coupling & PISO \\
Momentum & Second order upwind \\
Flow & laminar \\
\hline
\end{tabular}

\section{Experiment Purpose}

Considering the immune isolation, carrier requirements, shear tolerance and other factors of MSCs and EPCs, the project designed immunoisolated perfused co-culture bioreactor (IPCB, shown in Fig.1) for two types of different cells. The dynamic perfusion environment provided by the upper and lower bi-level of the culture chambers and immune isolation of intermediate polycarbonate diaphragm, combined with calcium alginate/gelatin microbead encapsulated NSCs and EPCs and other measures, can effectively integrate hypoxic environment to achieve NSCs efficient amplification purposes. The project will design and prepare polycarbonate diaphragm which have immune isolation and investigate diaphragm's flow field distribution, shear and dynamic pressure changes and mass transfer efficiency on both sides culture chamber by both simulation and experiment aspect.

\section{Results and Discussion}

Design and structure of IPCB

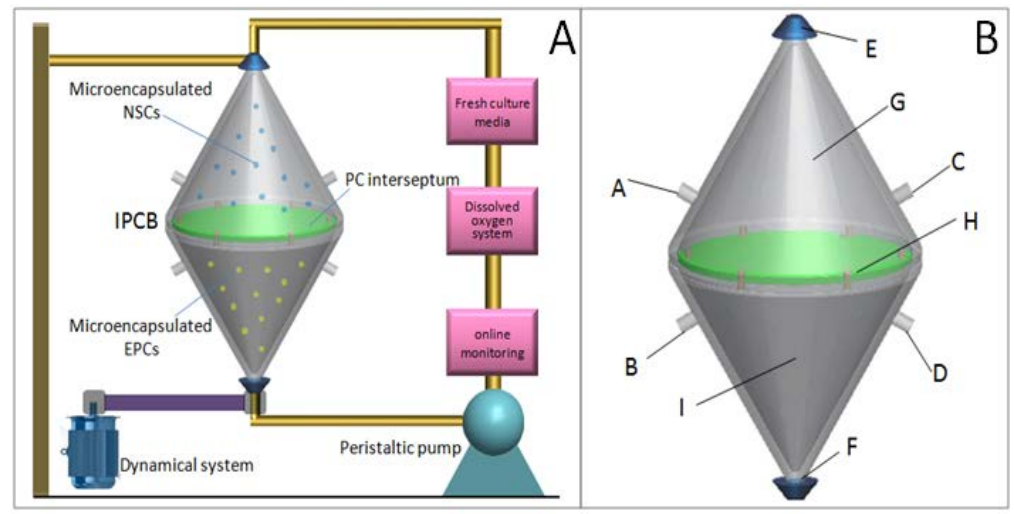

Fig.1 Schematic for structure and function of immnuoisolation perfusion co-culture bioreactor system and cell culture chamber. (A) Immnuoisolation perfusion co-culture bioreactor system; (B)

Cell culture chamber with up and down bi-level design.

In the IPCB, as shown in Fig.1B, A-D are sampling ports, $\mathrm{E}$ is the medium outlet of perfusion circuit, $F$ is the medium entrance of perfusion circuit, $G$ is the upper culture chamber, which will used for culture of calcium alginate/gelatin microbeads embedded with NSCs; I is the lower culture chamber, which will used for culture of calcium alginate/gelatin microbeads embedded with EPCs; Between two layers of polycarbonate diaphragm is $\mathrm{H}$, which can be used for immune isolation of dynamic co-cultured NSCs and EPCs.

Flow field simulation of IPCB

In Fig.2, A is model grid distribution of three-dimensional dynamic reactor system of IPCB. 
Distribution of microbeads in X-axis direction of the upper and lower bi-level culture chambers is shown in Fig.2B, in the dynamic culture process (50rpm speed, rotate along the $\mathrm{Z}$ axis), we can see that most of NSCs embedded in the micro plastic beads of upper culture chamber are in the middle of the polycarbonate diaphragm, a precious few of them located at the top, however, distribution of micro plastic beads in the down culture chamber are more scattered. $\mathrm{C}$ is the dynamic pressure distribution in the left side of cultivation chamber.

As can be seen from Fig.2D, the hydrostatic pressure distribution of IPCB's upper and lower levels in culture chambers is more evenly distributed; as medium speed in culture chamber, the speed of the regions nears polycarbonate diaphragm and the upper and lower layers of the culture chamber wall surface are slightly higher, other regions are more balanced distributed.

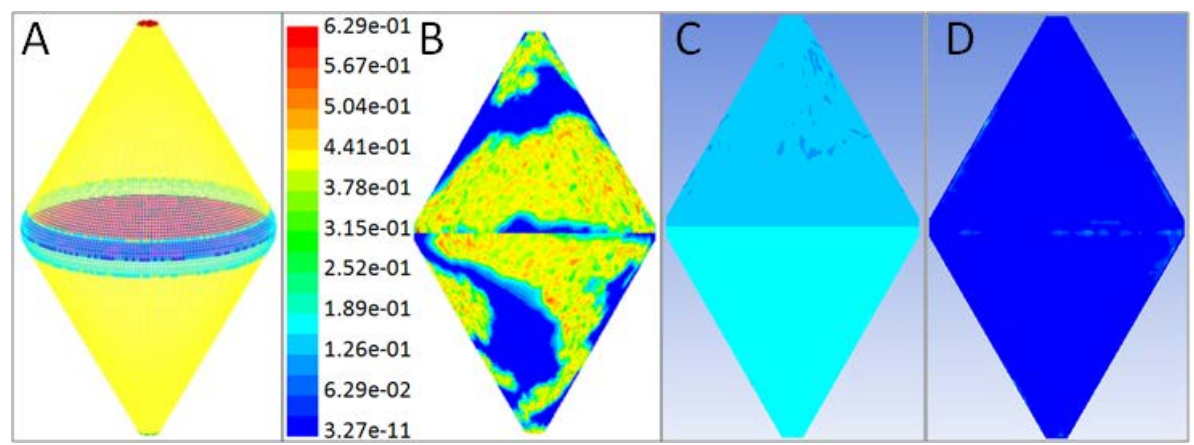

Fig.2 Simulation mesh distribution of flow field in IPCB and flow field analysis of up and down bi-level culture chamber.

\section{IPCB culture chamber flow field analysis}

Fig. 3 is a sectional distribution of up and down layer, $\pm 10 \mathrm{~mm}, \pm 20 \mathrm{~mm}, \pm 30 \mathrm{~mm}$ and $\pm 40 \mathrm{~mm}$ based on polycarbonate membrane. Dynamic pressure distribution of cell plastic beads surface in IPCB culture chamber of upper and lower levels (A). Dynamic pressure distribution(C) and velocity distribution(D) of medium. Viewing the flow field analysis from the section of upper and lower levels(Fig.3A), plastic beads in the cell wall surface have the higher dynamic pressure value and have lower value in the central region; The results of cell distribution are showed on Fig.3B, plastic beads NSCs are mostly distributed near the polycarbonate membrane in the middle, a small part is at the top; And plastic beads are more scattered distribution in the lower level. Known from the dynamic analysis (Fig.3C), the position of polycarbonate membrane in the middle has the minimum value of the dynamic pressure, the remaining parts have the greater value because of the perfusion medium can eliminate the difference between the gravity of micro plastic beads and the buoyancy of medium. And the velocity of the edges, the top and the bottom have the greater value(Fig.3D), because the velocity distribution is the same as Fig.3C.

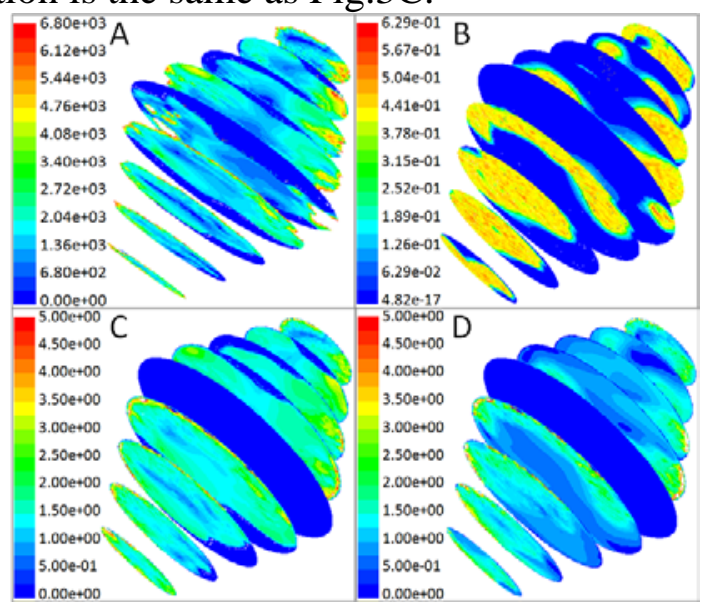

Fig.3 low field analysis of up and down bi-level culture chamber of IPCB. Sectional distribution of up and down layer, $\pm 10 \mathrm{~mm}, \pm 20 \mathrm{~mm}, \pm 30 \mathrm{~mm}$ and $\pm 40 \mathrm{~mm}$ based on polycarbonate membrane. Dynamic pressure distribution (A) of surface on microbeads cultured in bi-level, the distribution of the cultured NSCs and EPCs (B), dynamic pressure distribution of the medium (C) and the velocity distribution (D) of up and down bi-level. 


\section{Conclusion}

In summary, the micro plastic beads are more evenly distributed under perfusion conditions, in favor of the mass transfer. The velocity of medium is slightly larger near polycarbonate membrane and the culture chamber wall surface, and has more balanced distribution of other regions, thus greatly reducing the shear. Static pressure and dynamic pressure of the culture chamber are generally evenly distributed, providing a stable environment to the neural stem cell proliferation.

\section{Acknowledgement}

This work was supported by the Fok Ying Tung Education Foundation (132027), National Natural Science Foundation of China (31370991/31170945), the State Key Laboratory of Fine Chemicals (KF1111), the Joint Open Foundation of Natural Science Foundation of Liaoning and Shenyang National Laboratory for Materials Science (2015021017) and the Fundamental Research Funds for the Central Universities (DUT14YQ106/DUT15QY47/16ZD210) and SRF for ROCS, SEM.

\section{References}

[1] Parent JM, Anderson SA. Reprogramming patient-derived cells to study the epilepsies [J]. Nat Neurosci. 2015; 18(3): 360-366.

[2] Otsu M, Nakayama T, Inoue N. Pluripotent stem cell-derived neural stem cells: From basic research to applications [J]. World J Stem Cells. 2014; 6(5): 651-7.

[3] Butti E, Cusimano M, Bacigaluppi M, Martino G. Neurogenic and non-neurogenic functions of endogenous neural stem cells [J]. Front Neurosci. 2014; 8: 92.

[4] Aleynik A, Gernavage KM, Mourad YSh, Sherman LS, Liu K, Gubenko YA, Rameshwar P. Stem cell delivery of therapies for brain disorders [J]. Clin Transl Med. 2014; 3: 24.

[5] Choi KA, Hwang I, Park HS, Oh SI, Kang S, Hong S. Stem cell therapy and cellular engineering for treatment of neuronal dysfunction in Huntington's disease [J]. Biotechnol J. 2014; 9(7): 882-94.

[6] Ambasudhan R, Dolatabadi N, Nutter A, Masliah E, Mckercher SR, Lipton SA. Potential for cell therapy in Parkinson's disease using genetically programmed human embryonicstem cell-derived neural progenitor cells [J]. J Comp Neurol. 2014; 522(12): 2845-56.

[7] Ramasamy S, Narayanan G, Sankaran S, Yu YH, Ahmed S. Neural stem cell survival factors. Arch Biochem Biophys [J]. 2013; 534(1-2): 71-87.

[8] Deleyrolle LP, Reynolds BA. Isolation, expansion, and differentiation of adult Mammalian neural stem and progenitor cells using the neurosphere assay [J]. Methods Mol Biol. 2009; 549: 91-101.

[9] Moeller ML, Dimitrijevich SD. A new strategy for analysis of phenotype marker antigens in hollow neurospheres [J]. J Neurosci Methods. 2004; 139(1): 43-50.

[10]Sokolowski K, Obiorah M, Robinson K, McCandlish E, Buckley B, DiCicco-Bloom E. Neural stem cell apoptosis after low-methylmercury exposures in postnatal hippocampus produce persistent cell loss and adolescent memory deficits [J]. Dev Neurobiol. 2013; 73(12):936-49.

[11]Saurat N, Andersson T, Vasistha NA, Molnár Z, Livesey FJ. Dicer is required for neural stem cell multipotency and lineage progression during cerebral cortex development [J]. Neural Dev. 2013; 8: 14.

[12]Chiang MC, Lin $\mathrm{H}$, Cheng $\mathrm{YC}$, Yen $\mathrm{CH}$, Huang RN, Lin KH. $\beta$-adrenoceptor pathway enhances mitochondrial function in human neural stem cells via rotarycell culture system [J]. J Neurosci Methods. 2012; 207(2): 130-6. 
[13]Vissapragada R, Contreras MA, da Silva CG, Kumar VA, Ochoa A, Vasudevan A, Selim MH, Ferran C, Thomas AJ.

Bidirectional crosstalk between periventricular endothelial cells and neural progenitor cells promote s theformation of a neurovascular unit [J]. Brain Res. 2014; 1565: 8-17.

[14]Rosell A, Morancho A, Navarro-Sobrino M, Martínez-Saez E, Hernández-Guillamon M, Lope-Piedrafita S, Barceló V, Borrás F, Penalba A, García-Bonilla L, Montaner J. Factors secreted by endothelial progenitor cells enhance neurorepair responses after cerebral ischemia in mice [J]. PLoS One. 2013; 8(9):e73244. 